\title{
Growth textures of thick sputtered films and multilayers assessed via synchrotron transmission Laue
}

\author{
M. A. Vill \\ Department of Materials Science and Engineering, University of Michigan, Ann Arbor, \\ Michigan 48109-2136 \\ Z. U. Rek \\ Stanford Synchrotron Radiation Laboratory, Stanford, California 94305 \\ S. M. Yalisove and J. C. Bilello ${ }^{\text {a) }}$ \\ Department of Materials Science and Engineering, University of Michigan, Ann Arbor, \\ Michigan 48109-2136
}

(Received 17 January 1995; accepted for publication 26 May 1995)

The growth textures of thick sputtered Mo metallizations and Mo/W multilayers, were characterized via a synchrotron white-beam (WB) $\mathrm{x}$-ray transmission Laue technique. Transmission $\mathrm{x}$-ray diffraction studies of Mo specimens up to $61 \mu \mathrm{m}$ thick were performed with WB synchrotron radiation; while the practical thickness limit for similar observations using a conventional laboratory $\mathrm{Cu} K(\alpha) \mathrm{x}$-ray source is ten times smaller. This unique approach used polychromatic $\mathrm{x}$ rays to simultaneously produce diffraction from a wide spread of orientations of many crystallographic planes for all the grains within a relatively large specimen volume $\left(\approx 60 \times 10^{6} \mu \mathrm{m}^{3}\right)$. These patterns were obtained for polycrystalline 31 - and $61-\mu \mathrm{m}$-thick $\mathrm{Mo} / \mathrm{W}$ multilayer specimens, and a 35- $\mu$ m-thick-monolithic Mo foil specimen. In all three cases the alignment of specimen grains was similar to what would be expected for single-crystal transmission patterns, except that the recorded intensity distributed was less localized. The WB transmission images were indexed using a reciprocal space construction for the Laue case. In the multilayers, the grains were oriented out-of-plane such that $\langle 110\rangle$ crystallographic planes were aligned in the direction of sputter growth, while in the monolithic Mo specimen 〈111〉 crystallographic planes were so aligned, i.e., perpendicular to the deposition substrate. A spread in orientation of $\sim 5^{\circ}$ was measured in the multilayer specimens, while the monolithic Mo specimen showed a spread of $\sim 30^{\circ}$ when compared to a perfect single-crystal orientation. Preferred orientation was also observed within the plane of growth to varying degrees for all three samples. C. 1995 American Institute of Physics.

\section{INTRODUCTION}

Characterization of preferred crystallographic orientation (texture) is necessary to understand the properties of anisotropic materials, because they are strongly dependent upon crystallographic direction. This is particularly true in the case of vapor deposits which often have large preferentially oriented and shaped grains. ${ }^{1,2}$ For this reason characterization of texture and crystallographic structure has been the topic of many recently reported thin film and multilayer studies. In these previous studies a variety of techniques have been used, including surface $\mathrm{x}$-ray diffraction techniques, ${ }^{3-5} \mathrm{x}$-ray fluorescence, ${ }^{5}$ scanning electron microscopy (SEM), ${ }^{6}$ transmission electron microscopy (TEM), ${ }^{5}$ and reflection highenergy electron diffraction (RHEED). ${ }^{4}$ Each of these techniques relates information about structure and texture, but depth sensitivity is limited.

Texture is usually determined by measuring $x$-ray reflection and/or transmission pole figures. These traditional techniques use laboratory $\mathrm{x}$-ray sources and, therefore for transmission experiments, require samples that are thinner than the extinction depth of the $\mathrm{x}$-ray wavelength of the material. On the other hand, reflection methods only assess the surface

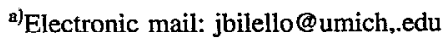

region of material, i.e., approximately half the sample thickness, or less.

In this work, transmission Laue $^{7}$ texture studies were performed using polychromatic x-ray radiation from a synchrotron source with a detector set-up similar to one employed by Stock et al. ${ }^{8}$ In the present work, diffraction patterns were recorded on photographic film, which was then interpreted using reciprocal space mapping to determine the growth direction and spread in the preferred orientation of the sample. The high energy and intensity of a synchrotron source made transmission through thick specimens, combined with reasonable recording times, convenient. Finally, observations of the spread in orientation, both radial and axial to the growth direction, is made possible by the use of polychromatic radiation.

It is important to note that the preferred orientations identified are not simple fiber textures, which are determined from Debye rings, a result of monochromatic transmission Laue diffraction. ${ }^{9}$ The monochromatic method can only identify the presence or lack of a fiber texture, while the technique used in this work is a straightforward method of quickly determining both in-plane and out-of-plane alignment, as well as the spread in the preferred orientation. 


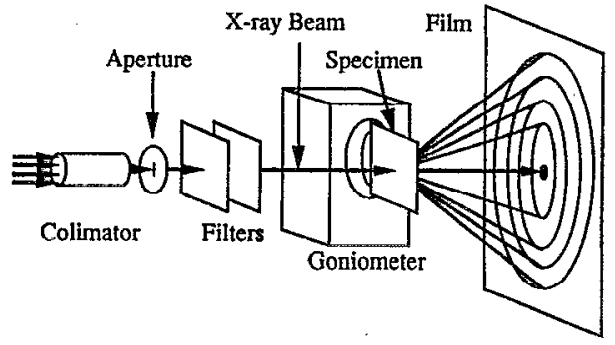

FIG. 1. White-beam synchrotron transmission Laue experimental set-up.

\section{EXPERIMENT}

Three types of specimens were evaluated: a $35-\mu \mathrm{m}$-thick monolithic Mo film, a 31- $\mu$ m-thick Mo/W multilayer, and a $61-\mu \mathrm{m}$-thick Mo/W multilayer. All were produced by planar magnetron sputter deposition using the same conditions, which have been previously reported. ${ }^{10}$ Both the monolithic Mo film and the 31- $\mu$ m-thick multilayer were deposited onto the as-received polished surface of a $\mathrm{Si}(100)$ wafer. The 61 $\mu \mathrm{m}$-thick multilayer was deposited onto the unpolished back surface of the wafer.

The preparation details for the multiscalar multilayers thick specimens have been described elsewhere. ${ }^{11}$ Briefly, these samples were made by combining thick monolithic Mo layers $(5 \mu \mathrm{m})$, with a hard stack which had 29 alternating thin $(4 \mathrm{~nm})$ layers of $W$ and Mo and this process was repeated until the overall sample thickness was reached. The monolithic Mo layers comprised $97 \%$ of the specimen volume.

Texture investigations were performed using two methods: White-beam synchrotron transmission Laue diffraction patterns were observed to determine the through thickness texture, and pole figures were also recorded, using the more traditional Schulz reflection geometry using a lab source. These results are compared below.

Transmission Laue patterns were imaged on Kodak SR-5 $\mathrm{x}$-ray photographic film at beamline II-2 at Stanford Synchrotron Radiation Laboratory (SSRL), with the experimental components aligned in the traditional pinhole configuration, ${ }^{12,13}$ as shown in Fig. 1. The primary $x$-ray beam exited the synchrotron storage ring through a bcryllium window, was collimated by a 1-mm-diameter round aperture, and passed through $\mathrm{Nb}$ and $\mathrm{Zr}$ sheet filters before impinging on the specimen. The primary beam was filtered to narrow the wavelength spectrum to various ranges in order to probe reciprocal space, as will be explained in Appendix I. The photographic plate was placed behind the specimen at a sample-to-film distance (SFD) of $74 \mathrm{~mm}$.

In this transmission Laue work, each specimen was aligned with its growth axis parallel to the incident primary beam, in order to identify the growth direction. Later, each specimen was rotated and diffraction patterns were recorded for different incident beam orientations to confirm the growth direction and other texture information. Specimen rotation was made possible by mounting each on a Huber goniometer that provided two translational and three rotational degrees of freedom. This allowed for precise alignment of the specimen with respect to the incident beam.

Reflection pole figures corresponding to $\{110\},\{200\}$, and $\{211\}$ planes were recorded from the growth initiation and growth termination surfaces of each specimen, so that the textures indicated by the transmission Laue patterns could be compared to the standard laboratory technique. They were recorded over a tilt range of $90^{\circ}-15^{\circ}$, using the Schulz geometry on a Rigaku powder camera with texture attachments and a $12 \mathrm{kw}$ Rigaku Rotaflex rotating anode $\mathrm{Cu}$ target. Results are reported below using the times-random representation. ${ }^{14}$ Pole figures are presented from only the $61-\mu \mathrm{m}$-multilayer specimen, because they provide additional information, while pole figures taken for the other two specimens simply confirm the indications of the transmission Laue patterns.

\section{RESULTS}

\section{A. Transmission Laue}

The data presented in Figs. 2-4 report the white-beam transmission Laue diffraction patterns recorded using the previously mentioned conditions $(\mathrm{Nb}$ and $\mathrm{Zr}$ filters and the primary beam aligned parallel to the specimen growth direction). Growth normals of all samples were found to deviate from the specimen normal by an angle of between $2^{\circ}$ and $10^{\circ}$.

The diffraction pattern produced by the $31-\mu \mathrm{m}$-thick Mo/W multilayer, Fig. 2(a), consists of a series of faint intensity circles superimposed on a group of strong intensity concentrations that display twofold rotational symmetry. The circles (or rings) result from a superposition of the absorption edges of the filters (plus the sample itself) and the polychromatic Bragg diffraction from the polycrystalline film. These diffraction-absorption (DA) rings have been described in detail elsewhere. ${ }^{15}$ Two are strong ( $a$ and $c$ ) and others are weak. The two strong ones can be identified as $\{110\}$ and $\{211\}$ rings from Bragg Law. A total of 26 intensity concentrations were visible in the original pattern. These are indexed in the schematic displayed in Fig. 2(b). This indexing was performed in the following manner. The intensity concentrations were identitied in order, starting from small radii (a) and working out to the large radii circles. Comparing Figs. 2(a) and 2(b): Inside the first strong DA ring are two intensity concentrations arranged vertically that stem from diffraction by $\{110\}$ planes and two arranged horizontally that stem from diffraction by $\{200\}$ planes. Adjacent to the first strong DA ring are two strong $\{110\}$ concentrations arranged vertically. Radiating from those are four more that are separated from each other by $\sim 23^{\circ} / 157^{\circ}$ and are composed of diffraction from $\{321\}$ and $\{431\}$ planes. Two more sets of four intensity concentrations are seen at about the same radius and arranged vertically; they are produced by diffraction from $\{301\}$ and $\{211\}$ planes. Those from the $\{310\}$ planes are separated by angles of $\sim 26^{\circ} / 154^{\circ}$ and those from the $\{211\}$ planes are separated by $\sim 71 \% 109^{\circ}$. Adjacent to the second strong DA ring is a set of four strong $\{211\}$ images arranged vertically that are separated by angles of $\sim 48^{\circ} / 132^{\circ}$. At the edge of the original figure was another set of four faint concentrations that are out of the field of view in the final repro- 


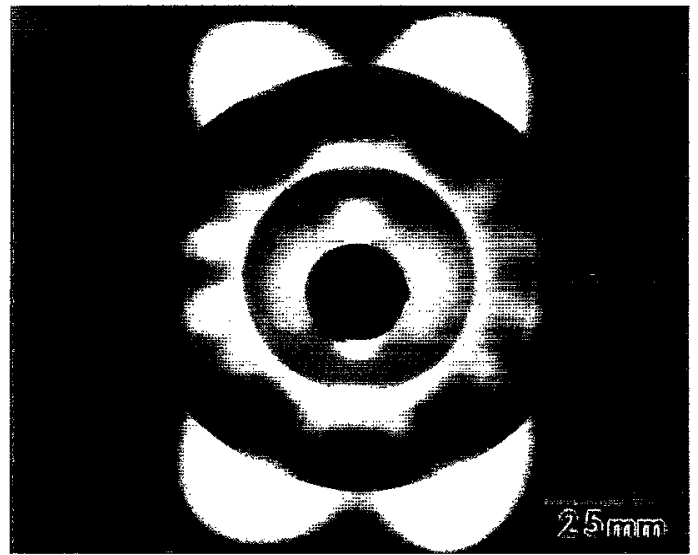

(a)

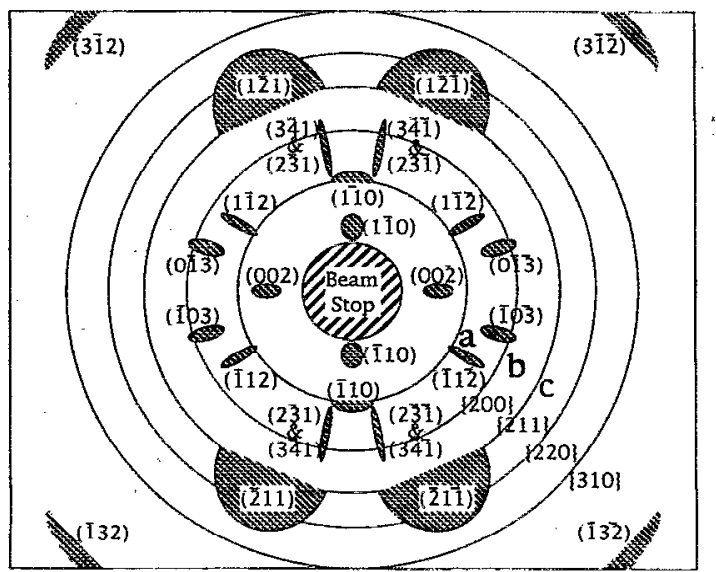

(b)

FIG. 2. (a) White-beam transmission Laue pattern of a $31-\mu$ m-thick multiscalar multilayer, recorded using a primary beam that had been filtered through $117 \mu \mathrm{m} \mathrm{Z} \mathrm{Z}$ and $40 \mu \mathrm{m} \mathrm{Nb}$ sheet. (b) A schematic representation of the diffraction pattern with all diffracted intensity concentrations indexed. Polychromatic DA rings seen in the diffraction pattern are labeled $a-c$ and indexed in the schematic. This pattern displays a two-fold rotational symmetry. Azimuthal rotations are measured as rotations about the pattern center, and inclination angles are measured as radial distances from the pattern center.

duction in Fig. 2(a). The intensity concentrations are separated by $\sim 65^{\circ} / 115^{\circ}$, and result from diffraction by $\{321\}$ planes. The observed angular spread is most easily measured in the four strong $\{211\}$ concentrations and is $\sim 5^{\circ}$ in both inclination and azimuth. Inclination is measured radially out from the center and azimuth is measured rotationally about the center of both transmission Laue diffraction patterns and pole figures. In pole figures inclination is denoted by the symbol " $\beta$ " and azimuth is indicated by the symbol " $\alpha$."

The diffraction pattern produced by the $61-\mu \mathrm{m}$-thick Mo/W multilayer, Fig. 3, consists of a series of strong DA rings superimposed on a group of strong intensity concentrations that display twofold rotational symmetry. Five strong rings (a-e) are clearly visible in Fig. 3, with portions of another. They result from diffraction by $\{110\},\{200\},\{211\}$, $\{220\},\{222\}$, and $\{321\}$ planes, respectively. Indexing of DA rings will be addressed in the appendix. A total of 21 inten-

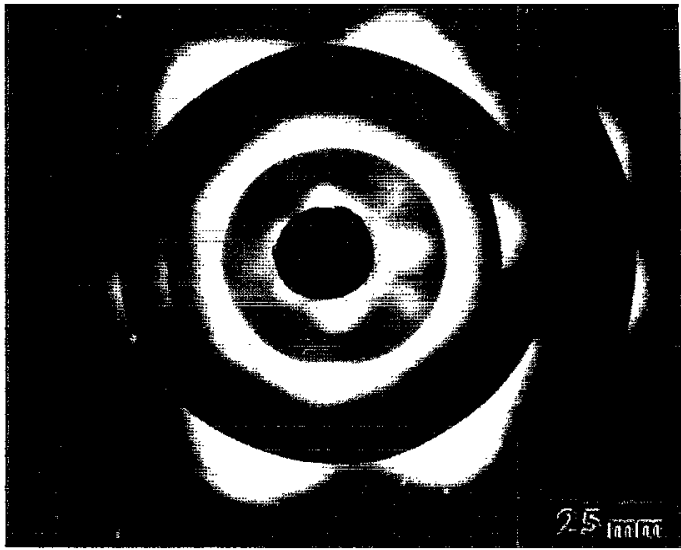

(a)

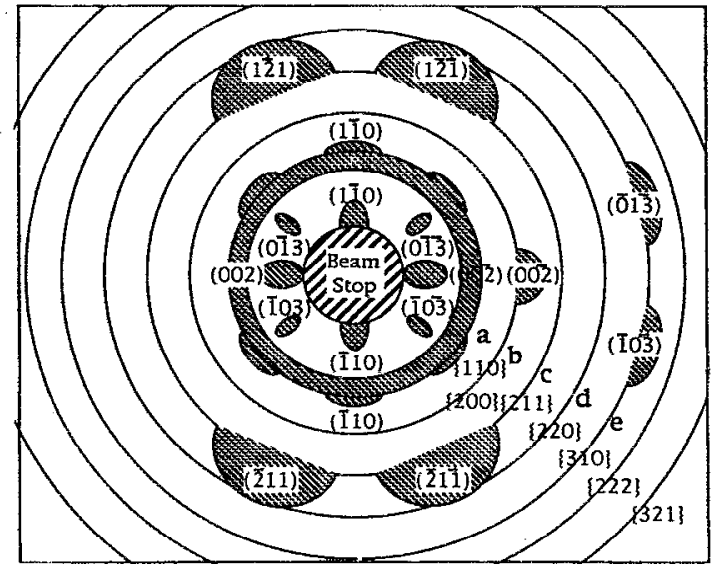

(b)

FIG. 3. (a) White-beam transmission Laue pattern of a $61-\mu$ m-thick multiscalar multilayer, recorded using a primary beam that had been filtered through a $117 \mu \mathrm{m} \mathrm{Zr}$ and $40 \mu \mathrm{m} \mathrm{Nb}$ sheet. (b) A schematic representation of the diffraction pattern with all diffracted intensity concentrations and DA rings indexed. Visible DA rings are labeled $\mathrm{a}-\mathrm{d}$.

sity concentrations are visible in the pattern in Fig. 3(a). Intensity concentrations are identified in order, again, from small radii a to large radii e. As above with Fig. 2, the indexing of Fig. 3(a) is illustrated in the schematic in Fig. 3(b). The ratio of measured intensity between the strong and weak portions of the DA rings is $\sim 9: 1$ as determined by microdensitometry, and again the angular spread is $\sim 5^{\circ}$, in both inclination and spread.

Similarly the diffraction pattern produced by the $35-\mu \mathrm{m}$ thick monolithic Mo specimen, Fig. 4(a) has been indexed as shown in Fig. 4(b). The especially bright intensity concentration with two horizontal lobes seen in the center of Fig. 4(a) is attributed to two sources, namely, $\{110\}$ diffraction from the sample superimposed with the diffuse scattering from the direct transmitted beam. The remaining intensity lobes are indexed in Fig. 4(b) and need not be described further. Finally, note an observed spread of $\sim 25^{\circ}$ in the intensity concentrations of this pattern, i.e., the $(\overline{22} 2)$ lobe shown schematically in Fig. 4(b). 


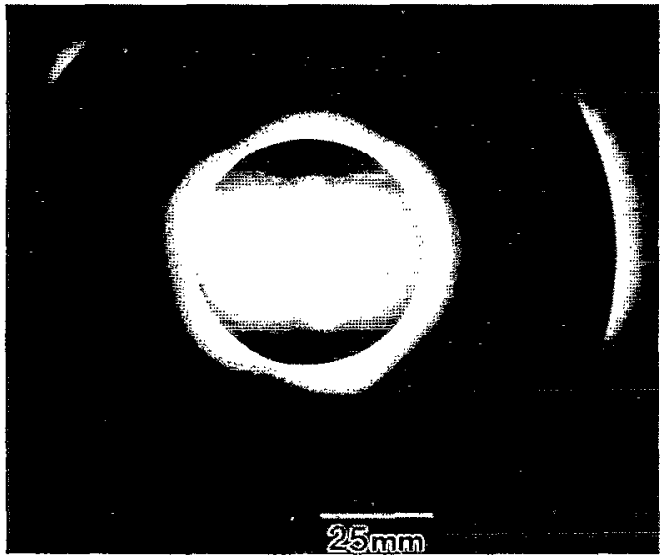

(a)

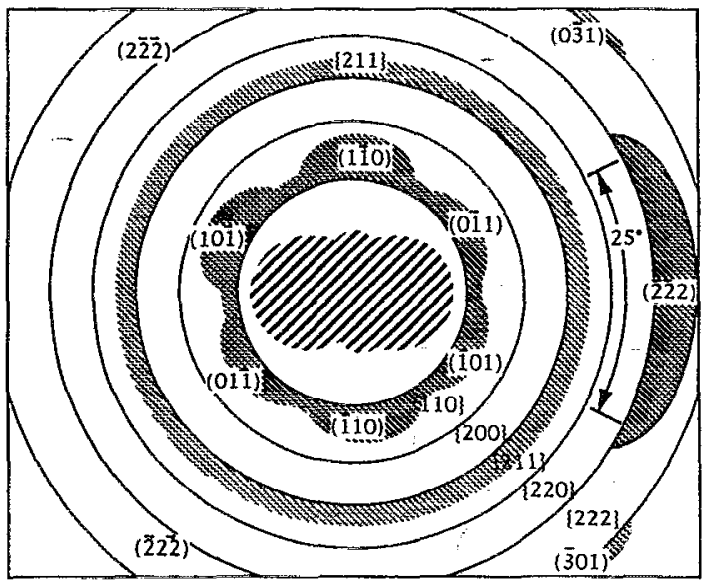

(b)

FIG. 4. (a) White-beam transmission Laue pattern of a $35 \mu \mathrm{m}$ monolithic Mo foil, recorded using a primary beam that had been filtered through 117 $\mu \mathrm{m} \mathrm{Zr}$ and $40 \mu \mathrm{m} \mathrm{Nb}$ sheet. (b) A schematic representation of the diffraction pattern with all diffracted intensity concentrations labeled.

\section{B. Pole figures}

Figure $5(\mathrm{a})$, is a $\{110\}$ diffraction vector pole figure taken on the growth termination surface of the $61 \mu \mathrm{m} \mathrm{Mo/W}$ multiscalar sample via a lab source with texture camera in the Schulz reflection geometry. Five $\{110\}$ peaks resulted with one located at the center of the stereographic projection $(\beta$ $=90^{\circ}$ ) and four are located on the $\beta=30^{\circ}$ circle. The four are separated azimuthally by angles of $60^{\circ} / 120^{\circ}$. They display an average spread of $5^{\circ}$, in both azimuth and inclination. This pole figure displays the same twofold rotational symmetry seen in the transmission diffraction pattern for the same sample (Fig. 3).

Figure $5(\mathrm{~b})$, is a $\{110\}$ diffraction vector pole figure taken on the growth initiation surface of the $61 \mu \mathrm{m} \mathrm{Mo/W}$ sample. The poles are located at $\beta \approx 57^{\circ}$ on the stereographic projection, just outside the $\beta=60^{\circ}$ circle, and possess a threefold rotational symmetry. They have an average inclination spread of $\sim 15^{\circ}$, and an average azimuth spread of $\sim 35^{\circ}$. In the bottom half of the stereographic projection, two

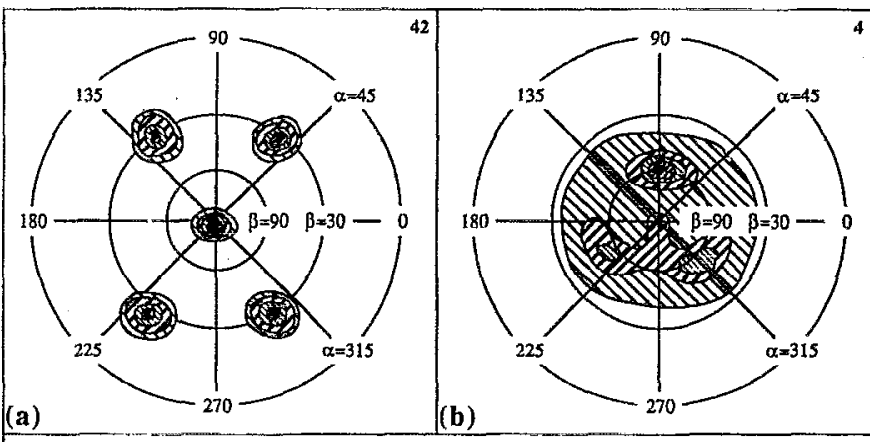

Relative Intensity: $90 \%$, 70\%, 50\%, $30 \%, \$ 10 \%$

FIG. 5. An evolution of texture is shown by $\{110\}$ pole figures of the two surfaces of the $61-\mu \mathrm{m}$-thick $\mathrm{Mo} / \mathrm{W}$ multilayer. (a) The twofold pattern of $\{110\}$ poles in the pole figure recorded for the growth termination surface indicates a $\langle 110\rangle$ growth direction, that is strongly aligned within the growth plane. (b) The threefold pattern of $\{110\}$ poles recorded for the initial growth surface indicates a $\langle 111\rangle$ growth direction that is not as strongly textured. The concentration that extends to the pattern center shows some significant presence of $\langle 110\rangle$ oriented grains, as well. Azimuthal angles are indicated by the symbol " $\alpha$ " and inclination angles are indicated by the symbol " $\beta$ ".

of the concentrations join together and extend to the center of the pattern, which will be discussed later.

\section{DISCUSSION}

Synchrotron transmission Laue data (Figs. 2 and 3, respectively) demonstrated that $\langle 110\rangle$ growth textures occurred for the 31- and $61-\mu \mathrm{m}$-thick multiscalar films while for the $35 \mu \mathrm{m}$ monolithic Mo film the preferred growth direction was $\langle 111\rangle$ (Fig. 4). These results were indicated by the timesrandom intensities of the $h k l$ pole at the center of the figures and this could be regarded as out-of-plane preferred orientation. However, instead of uniform random intensity being observed at larger azimuthal angles, rather marked preferential scattering pointed to a relatively well-developed in-plane texture as well. Hence all of the studied specimens possessed in-plane and out-of-plane alignment of crystallographic directions. In order to clarify how a detailed analysis of the preferred orientation indexing, and relative degree of texture, was made from the synchrotron Laue transmission patterns it is necessary to discuss the interpretation in terms of a simple reciprocal space model.

The reciprocal space representation ${ }^{16}$ of this experiment constructed to index these patterns is represented by four planes of the reciprocal lattice in Fig. 6. The important terms are presented here and the details are provided in the appendix. In reciprocal space crystallographic planes are represented by points. The Ewald sphere is superimposed on this lattice to represent diffraction conditions. Diffraction will occur from all crystallographic planes whose reciprocal lattice points are intersected (or nearly intersected) by the Ewald spherc. The Ewald sphere represents the incident and diffracted beams and is drawn centered on a line connecting the $\{110\}$ point and the origin. (For the multiscalar multilayers we begin with the presupposition that the incident direction is the [110] direction.) The reciprocal space planes that are oriented normal to it are commonly used for referencing dif- 

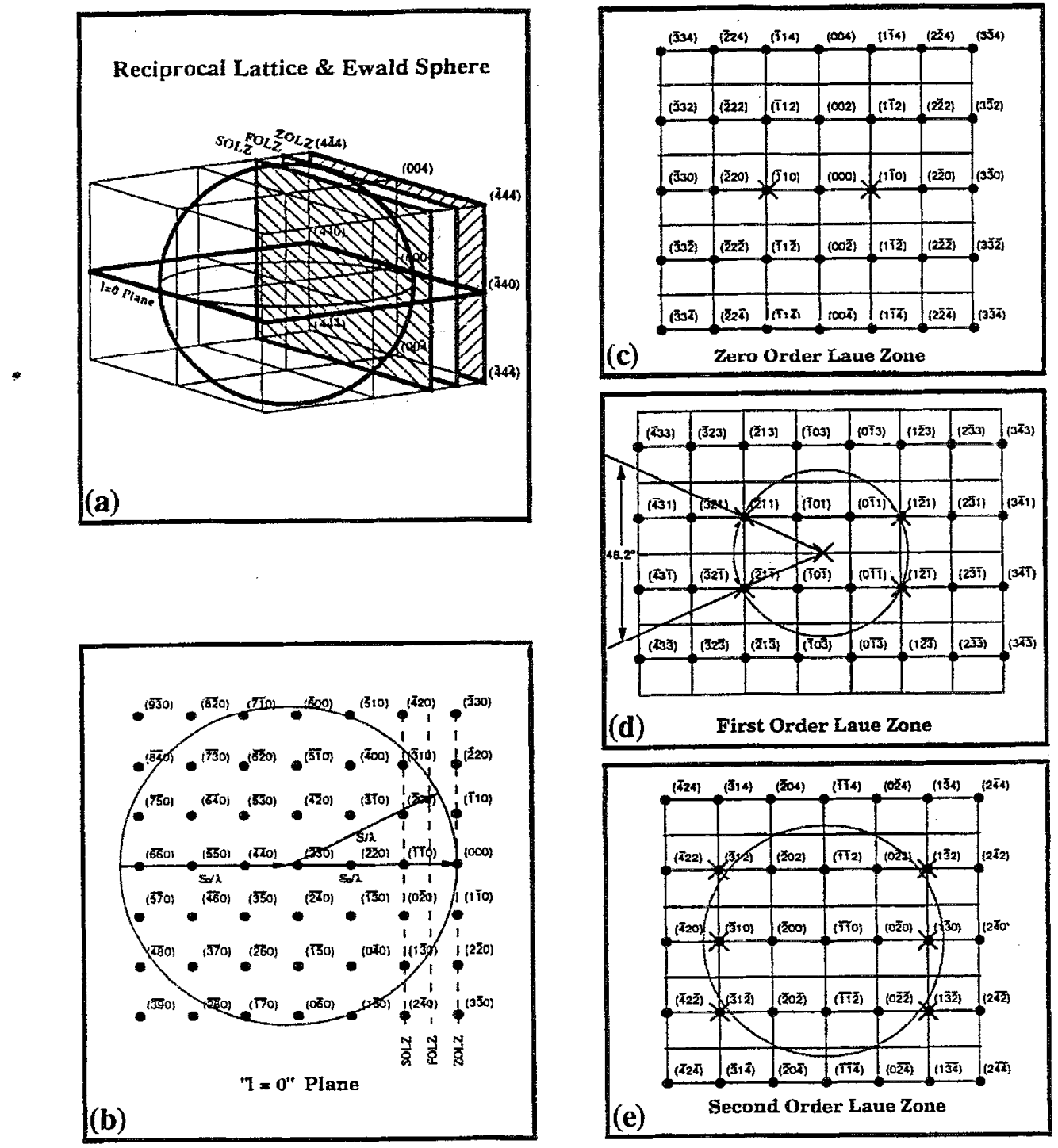

FIG. 6. "Reciprocal Space" construction for diffraction of $\lambda=0.735 \AA$ radiation by a Mo specimen. $\left(1 / a_{0(\mathrm{Mo})}=0.31735 \AA^{* 1}, 1 / \lambda=1.3605 \AA^{-1}\right)$. (a) Schematic representation of the reciprocal lattice and the Ewald sphere. (b) " $1=0$ " plane. (C) Zero order Laue diffraction zone. (D) First order Laue diffraction zone. (E) Second order Laue diffraction zone.

fracting crystallographic planes and are called Laue zones. They are shown in Fig. 6(a). The first of these intersects the Ewald sphere at the origin and is called the zero order Laue zone (ZOLZ). The next one to intersect the Ewald sphere is the first order Laue zone (FOLZ), and the second order Laue zone (SOLZ) is after that. Normally only the first two or three are required for indexing diffraction patterns.

The intensity concentrations seen in Fig. 4(a) are the result of diffraction of two spectral ranges of $x$ rays from crystal planes in the ZOLZ, FOLZ, and SOLZ. The Ewald spheres of the primary range intersected only the $(\overline{1} 10)$ and (110) plane points in the ZOLZ. Those of the secondary range intersected $(\overline{1} 10),(1 \overline{1} 0),(002),(00 \overline{2}),(\overline{1} 12),(1 \overline{1} 2)$, $(\overline{11})$, and $(1 \overline{12})$ plane points.

In the FOLZ, $(\overline{2} 11),(1 \overline{2} 1),(\overline{2} 1 \overline{1})$, and $(1 \overline{21})$ plane points were intersected by Ewald spheres of the primary spectrum. Reciprocal space points from many planes were intersected by Ewald spheres of the secondary spectrum. Diffraction from $\{310\},\{321\}$, and $\{431\}$ type planes are apparent in Fig. 2(b).

In the SOLZ, $(\overline{3} 12),(1 \overline{3} 2),(\overline{3} 1 \overline{2})$, and $(1 \overline{32})$ were intersected by Ewald spheres of the primary spectrum; $(\overline{3} 10)$ and $(1 \overline{3} 0)$ were not; and only reciprocal space points of high index planes were intersected by spheres of the secondary spectrum. These planes made no contribution to the recorded pattern, because their diffracted intensities were insignificant. This is indicated in Table I, which lists the calculated diffracted intensities for crystallographic planes in Mo.

The 31- $\mu \mathrm{m}$-thick multilayer diffraction pattern indicated an almost completely textured (complete lack of misoriented grains) specimen with a $\langle 110\rangle$ growth direction. This is evidenced by the large number of visible diffraction concentrations, particularly those produced by diffraction of the secondary range of the spectrum [Fig. 7(c)] from crystallo- 
TABLE I. White-beam intenity concentrations for a typical Mo/W MSML specimen.

\begin{tabular}{lcc}
\hline \hline $\begin{array}{l}\text { Crystallographic plane } \\
(h k l)\end{array}$ & Spectral range & $\begin{array}{c}\text { Approximate } \\
\text { DFPC } \\
(\mathrm{mm})\end{array}$ \\
\hline$(\overline{1} 10),(1 \overline{1} 0)$ & Primary & $24 \mathrm{TIE}^{\mathrm{b}}$ \\
$(\overline{1} 21),(2 \overline{1} 1),(\overline{1} 2 \overline{1}),(2 \overline{\mathrm{I}})$ & Primary & $46 \mathrm{TIE}^{\mathrm{b}}$ \\
$(\overline{1} 32),(3 \overline{1} 2),(\overline{1} 3 \overline{2}),(3 \overline{1})$ & Primary & $84 \mathrm{TIE}^{\mathrm{b}}$ \\
$(\overline{1} 10),(1 \overline{1} 0)$ & Secondary & $7.5 \mathrm{TC}^{\mathrm{c}}$ \\
$(002),(00 \overline{2})$ & Secondary & $15 \mathrm{TC}^{\mathrm{c}}$ \\
$(\overline{103}),(0 \overline{1} 3),(\overline{1} 0 \overline{3}),(0 \overline{13})$ & Secondary & $31 \mathrm{TC}^{\mathrm{c}}$ \\
$(\overline{1} 12),(1 \overline{1} 2),(\overline{1} 1 \overline{2}),(1 \overline{12})$ & Secondary & $25 \mathrm{TC}^{\mathrm{c}}$ \\
$(\overline{4} 1),(3 \overline{4} 1),(\overline{4} 3 \overline{1}),(3 \overline{1})$ & Secondary & $36 \mathrm{TC}^{\mathrm{c}}$ \\
\hline
\end{tabular}

${ }^{3} \mathrm{DFPC}$-distance from the pattern center. $\alpha$

"TIE-measured to the inside edge of the ring.

'TC-measured to the center of the ring.

graphic planes located in the FOLZ of the reciprocal space representation [Fig. 6(d)]. The specimen must be highly textured to produce those concentrations in the pattern for two reasons. First, the peak intensity of the secondary region of the spectrum is far less than that of the primary region, and second, the diffracted intensity of $\{310\}$ planes, for example, is far less than that of $\{110\}$ planes for the same incident intensity, as shown in Table I. The specimen texture is best expressed in growth terminology as having an in-plane and out-of-plane texture, where the plane refers to the growth plane. This is because the majority of the grains in the specimen have common crystallographic directions aligned. In addition, the absence of intense, continuous DA rings from the diffraction pattern, and the complete absence of certain rings denotes the absence of randomly oriented grains. Although, the continuous DA rings from the more intensely diffracted planes indicate the presence of an azimuthal texture, which denotes grains that have one crystallographic direction aligned, but are aligned randomly about that direction. The grains are said to be aligned out-of-plane, but not in-plane, because the alignment axis corresponds to the growth axis. All angular and linear relationships of the observed intensity concentrations are accounted for by the reciprocal space construction, shown in Fig. 6.

Analysis of the white-beam transmission patterns of the $61 \mu \mathrm{m}$ multilayer and the monolithic Mo specimens (Figs. 3 and 4) followed the same approach. The SFD for the dis- played patterns were 75.3 and $80.6 \mathrm{~mm}$, respectively. The difference in SFD accounts for the slight difference observed between the corresponding DA ring radii of the three patterns. The same reciprocal space construction that was used twice alrcady was used again for the $61 \mu \mathrm{m}$ multilayer, since it had a Mo lattice and a $\langle 110\rangle$ growth direction. However, the monolithic Mo had a $\langle 111\rangle$ growth direction, as indicated by the threefold rotational symmetry seen in the transmission pattern. Therefore, a slightly different reciprocal space representation was required. It employed the same lattice spacing and Ewald spheres, but had a different incident beam direction and different zones. The results of this indexing from analysis of the ZOLZ, FOLZ, and SOLZ have been shown in Fig. 3(b). The texture of the $61-\mu \mathrm{m}$-thick multilayer was further addressed using $x$-ray reflection pole figures, which showed a well developed $\langle 111\rangle$ growth texture on the initial growth surface, and a well developed $\langle 110\rangle$ growth texture on the final growth surface [Figs. 5(a) and 5(b)]. "In-plane" and "out-of-plane" alignment is apparent in both and can be confirmed by comparison with standard $\langle 111\rangle$ and $\langle 110\rangle$ stereographic projections. Together, the transmission and pole figure data indicate the evolution from a well-developed $\langle 111\rangle$ growth to a well-developed $\langle 110\rangle$ growth, with two important features.

The monolithic Mo diffraction pattern displayed a threefold texture in $\{110\}$ and $\{211\}$ concentration patterns surrounding the DA rings associated with the same planes (Fig. 4). From this we conclude that preferred growth was along a 〈111) direction. The near uniformity observed in the $\{211\}$ ring of Fig. 4 was caused by overlapping contributions from two groups of $\{211\}$ planes, one in the ZOLZ and one in the FOLZ of the reciprocal space representation of this diffraction condition (not shown). Nearly all grains are aligned in the preferred orientation, which is evidenced by the absence of intense continuous DA rings that could be attributed to randomly oriented grains, and by the high contrast between strong and weak regions of the pattern. An orientation spread (both inclination and azimuthal) of $\sim 25^{\circ}$ indicated a widely distributed texture.

It should also be noted that orientation distribution functions (ODFs) were calculated for the materials studied in this work using PopLA ${ }^{17}$ software. The results of that exercise confirm the physical ideas presented within this document. Therefore those results were omitted for the sake of brevity.
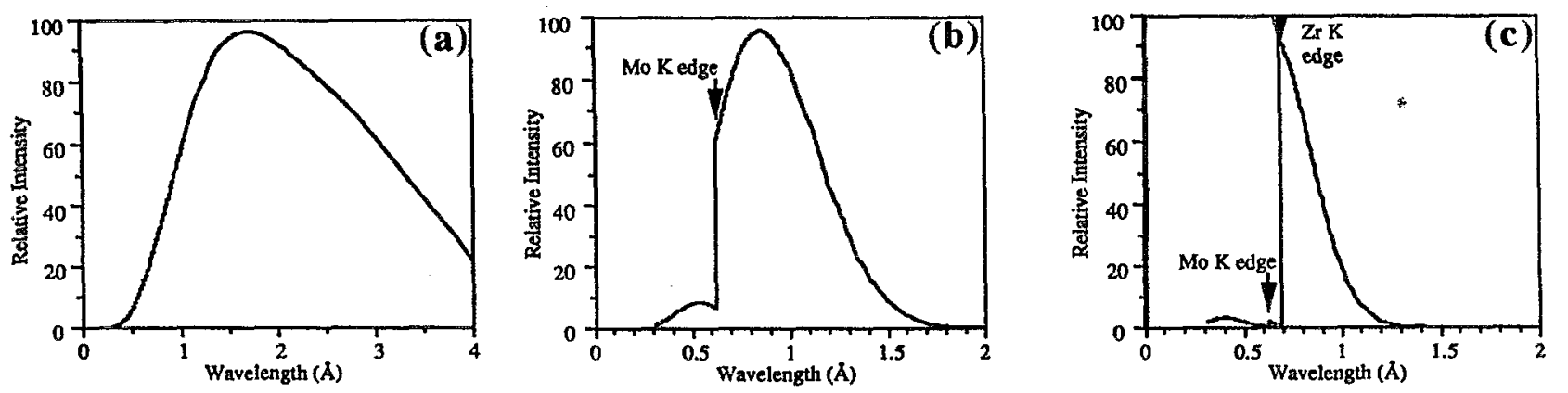

FIG. 7. X-ray spectra for various experimental conditions. (a) Incident beam. (b) Beam transmitted through a 31- $\mu$-thick Mo/W multilayer specimen. (c) Beam transmitted through a $31 \mu \mathrm{m} \mathrm{Mo/W}$ multilayer and $117-\mu \mathrm{m}$-thick $\mathrm{Zr}$ filter. 


\section{CONCLUSION}

A straightforward method has been shown to probe reciprocal space in thick, high $Z$ samples. White-beam synchrotron transmission Laue diffraction modified, by filters to control the diffraction, was used in the determination of the through-thickness texture of thick film metallizations and multilayers, which included measurement of azimuthal and inclination spread of grains in the sample.

Because the texture of thick films and multilayers can change as growth progresses, as demonstrated by the $61-\mu \mathrm{m}-$ thick multilayer in this study it is necessary to probe the entire thickness of these films to understand the evolution of these unusual morphologies. Transmission texture studies provide an indication of the through the thickness average texture, while standard methods can characterize texture only at the initial and final growth surfaces. With this method we have observed the dramatic shift in texture development caused by small additions of atoms. The addition of $<1.5$ at. $\% \mathrm{~W}$ to sputter deposited Mo films, in this multiscalar configuration, altered the texture from one having a $\langle 111\rangle$ growth direction to one having a $\langle 110\rangle$ growth direction.

\section{ACKNOWLEDGMENTS}

This work was supported by the USARO and ARPA under contract DAAL03-91-0235. The synchrotron work was conducted at the Stanford Synchrotron Radiation Laboratory, funded by the Department of Energy, Office of Basic Energy Sciences.

\section{APPENDIX}

All features observed in white-beam transmission patterns can be attributed to a combination of three effects: absorption of the incident beam, diffraction of the x-ray heam by specific crystallographic planes, and the texture of the specimen. Absorption reduces the spectrum of $x$ rays that is available for diffraction, which dictates what orientations of a family of crystallographic planes will diffract. Diffraction will occur for all crystallographic planes that satisfy the diffraction conditions defined by Bragg Law and reciprocal space. The texture of the specimen dictates which orientations of crystallographic planes that are available to diffract $x$ rays. In the absence of a preferred orientation (texture), only DA rings are present in the transmission pattern. Existence of a texture produces intensity concentration patterns in the circles.

The diffraction spectrum is dependent upon absorption by materials in the path of the primary beam. While the primary beam spectrum is continuous over a considerable range [Fig. 7(a)], the $K$ edges of filter materials separate that spectrum [Figs. 7(b) and 7(c)] into distinct ranges. In Fig. 7, calculated spectra ${ }^{18}$ are shown for three cases: (1) the primary beam, (2) the beam when transmitted through a 31 $\mu \mathrm{m}$-thick $\mathrm{Mo} / \mathrm{W}$ multilayer specimen with no prefilters, and (3) the beam when transmitted through a $34-\mu \mathrm{m}$-thick $\mathrm{Nb}$ filter, a $117-\mu \mathrm{m}$-thick $\mathrm{Zr}$ filter and a $31-\mu \mathrm{m}$-thick $\mathrm{Mo} / \mathrm{W}$ multilayer specimen. The spectrum of the primary beam is continuous from $\sim 0.5$ to $\sim 4.0 \AA$ with a peak at $\sim 1.7 \AA$. The augmented spectrum, produced by transmission through only

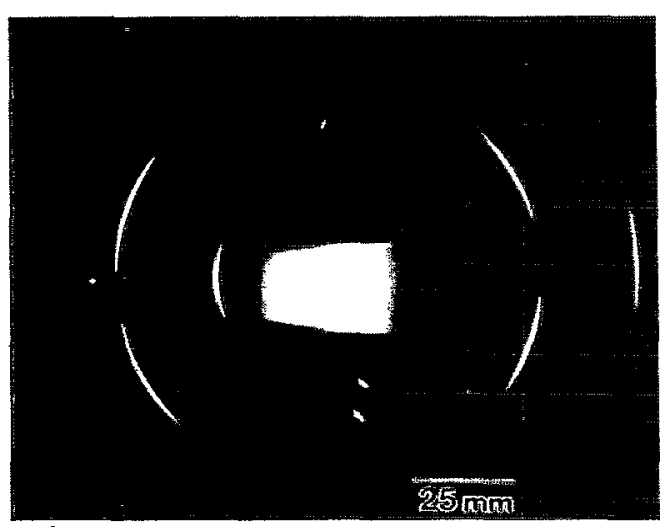

(a)

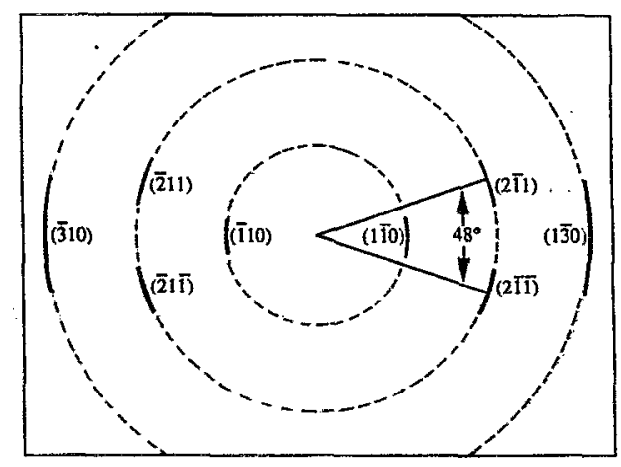

(b)

FIG. 8. (a) Monochromatic $(\lambda=0.735 \AA)$ transmission Laue pattern for 31$\mu \mathrm{m}$-thick Mo/W multilayer. (b) Schematic of the same pattern with intensity concentrations labeled with the name of the crystallographic plane that produced them.

the specimen [Fig. 7(b)] is separated by the Mo $K$ edge into two distinct ranges. The peak intensity of the primary range occurs at a wavelength of $\sim 0.75 \AA$, and is about 9.5 times as intense as the secondary spectrum. The spectrum produced by transmission through the specimen and the $\mathrm{Nb}$ and $\mathrm{Zr}$ filters [Fig. 7(c)] is separated by the Mo and $\mathrm{Zr} K$ edges into three ranges. The primary range extends from the $\mathrm{Zr} K$ edge $(0.69 \AA)$ to $\sim 1.20 \AA$, the secondary range extends from $\sim 0.30 \AA$ to the Mo $K$ edge $(0.61 \AA)$, and the tertiary range extends from 0.61 to $0.69 \AA$. Addition of the prefilters increased pattern contrast by narrowing the $\mathrm{x}$-ray spectrum, and thereby reducing overlap of diffracted concentrations. Transmission patterns produced with this combination of filters showed contributions from both the primary and secondary spectrum ranges.

Relative intensities were calculated from the first 40 (low index) possible diffracting planes, and the first 18 are listed in Table I, which displays the calculated intensity and the intensity observed in the transmission pattern seen in Fig. 2(a) using equations that can be found in many introductory $x$-ray texts. ${ }^{919}$ The calculation included consideration of the structure factor $(F)$, multiplicity factor $(p)$, and the Lorentzpolarization factor. For this material (Mo) and wavelength $(0.69 \AA)$, the relative intensity of $\mathrm{x}$ rays diffracted from planes having indices greater than $\{321\}$ is insignificant com- 
pared to that diffracted from $\{110\}$ planes (less than $2 \%$ ).

Reciprocal space constructions were employed in completion of the analysis. To facilitate ease of understanding this step was performed first for the monochromatic case and later extrapolated to the polychromatic case. A monochromatic transmission diffraction pattern [Fig. 8(a)] was recorded for the $31-\mu \mathrm{m}$-thick Mo/W multilayer using the same $\mathrm{SFD}$ and wavelength ( $\mathrm{Zr} K$ edge) employed in the whitebeam study. A schematic of the diffraction pattern is provided in Fig. 8(b). The SFD was set to $\sim 75 \mathrm{~mm}$ and a $\mathrm{Si}(111)$ monochromator was set to an angle of $\sim 6.4^{\circ}$ to produce an $\mathrm{X}$-ray wavelength of $\sim 0.7 \AA$. They were later confirmed using the radii of the identified DA rings and Bragg Law. The experimental values of wavelength $(0.735 \AA)$ and SFD $(78.9 \mathrm{~mm})$. The reciprocal space representation ${ }^{16}$ of this experiment constructed to index these patterns is represented by four planes of the reciprocal lattice in Fig. 6 . The Ewald sphere is drawn centered on a line connecting the $\{110\}$ point and the origin with a radius $\left(1.3605 \AA^{-1}\right)$.

For $x$ rays, the radius of curvature of the Ewald sphere is small, and in this case only the pair of spots that represent the (110) and (110) planes are near enough to $(000)$ to be intersected. Diffraction from them formed the intense, interior DA ring with two concentrations and $27 \mathrm{~mm}$ radius that is seen in Figs. 8(a) and 8(b). Because the Ewald sphere is tangent to the ZOLZ at the origin, no intersection circle is drawn in Fig. 6(b), and the (110) and (110) plane reciprocal space points are intersected because lattice distortion in the specimen causes these points to be stretched into spheres.

The intersection of the Ewald sphere with the FOLZ [Fig. 6(c)] forms a circle with a radius of $0.75 \AA^{-1}$. It passes through reciprocal space points representing $(\overline{2} 11),(2 \overline{1} 1)$, $(\overline{2} 1 \overline{1})$, and (211) planes. Diffraction from them formed the second DA ring [Figs. 8(a) and 8(b)] which has a radius of $52 \mathrm{~mm}$ and four intensity concentrations corresponding to the four diffracting planes. No other spots in the FOLZ were intersected by the Ewald sphere.
The intersection of the Ewald sphere with the SOLZ [Fig. 6(d)] forms a circle with a radius of $1.01 \AA^{-1}$. It passes very near spots representing two $\{310\}$ type planes and four $\{321\}$ type planes. The reciprocal space points are sufficiently distorted for all six to intersect the Ewald sphere. Diffraction from the $\{310\}$ type planes formed the third DA ring. The $\{321\}$ DA ring was too large to be included in the figure. Interpretation of all other synchrotron WB Laue transmission patterns followed by a similar analysis to the above.

${ }^{1}$ J. A. Thornton, Annu. Rev. Mater. Sci. 7, 239 (1977).

${ }^{2}$ C. R. M. Grovenor, H. T. G. Henzel, and D. A. Smith, Acta. Metall. 32, 773 (1984).

${ }^{3}$ O. Knotek, F. Loffler, and G. Kramer, Surf. Coat. Technol. 54, 241 (1992).

${ }^{4}$ F. Giron and P. Boher, Thin Solid Films 226, 9 (1993).

${ }^{5}$ W. W. Hoogenhof and T. W. Ryan, J. Magn. Mater. 121, 88 (1993).

${ }^{6}$ V. M. Fedosyuk and O. I. Kasyutich, Thin Solid Films 230, 4 (1993).

${ }^{7} \mathrm{M}$. von Laue, "Historical Introduction," International Tables for X-ray Crystallography, Volume 1 (Kynoch, Birmingham, England, 1952), pp. $1-5$.

${ }^{8}$ S. R. Stock, Y. H. Cheng, P. C. Huang, Z. U. Rek, and B. M. Ditchek, J. Appl. Phys. 73, 1737 (1993).

${ }^{9}$ D. B. Cullity, Elements of X-ray Diffraction (Addison-Wesley, Reading, MA, 1978).

${ }^{10}$ D. P. Adams, M. Vill, J. Tao, J. C. Bilello, and S. M. Yalisove, J. Appl. Phys. 74, 15 (1993).

${ }^{11}$ M. Vill, D. P. Adams, S. M. Yasilove, and J. C. Bilello, Acta. Metall. Mater. 43, 427 (1995).

${ }^{12}$ A. W. Hull, Phys. Rev. 10, 661 (1917).

${ }^{13} \mathrm{~J}$. L. Amoros, M. J. Buerger, and M. L. Canut de Amoros, The Laue Method (Academic, New York, 1974).

14 "Times random" scale is a convention for quantifying texture which appears in many references, one of which is C. S. Barrett and T. B. Massalski, Structure of Metals (McGraw-Hill, New York, 1966), pp. 541-567.

${ }^{15}$ M. Vill, S. G. Malhotra, Z. Rek, S. M. Yalisove, and J. C. Bilello, Material Research Society Symposium Proceedings (Materials Research Society, Pittshurgh, 1994), Vol. 317, p. 413.

${ }^{16} \mathrm{~K}$. W. Andrews, D. J. Dyson, and S. F. Keown, Interpretation of Electron Diffraction Patterns (Plenum, New York, 1967).

${ }^{17}$ PopLa, Orientation Distribution Software, Los Alamos Labs, University of California.

${ }^{18}$ S. Brennan and P. L. Cowan, Rev. Sci. Instrum. 63, 850 (1992).

${ }^{19}$ N. F. M. Henry, H. Lipson, and W. A. Wooster, The Interpretation of X-ray Diffraction Photographs (MacMillan, London, 1961). 